\title{
openheart Smokeless tobacco, snus, at admission for percutaneous coronary intervention and future risk for cardiac events
}

\author{
Ole Frobert (D) , ${ }^{1}$ Christian Reitan, ${ }^{2}$ Dorothy K Hatsukami, ${ }^{3}$ John Pernow, ${ }^{4}$ \\ Elmir Omerovic, ${ }^{5}$ Pontus Andell ${ }^{6}$
}

\begin{abstract}
- Additional material is published online only. To view please visit the journal online (http://dx.doi.org/10.1136/ openhrt-2019-001109).
\end{abstract}

To cite: Frobert 0 , Reitan $\mathrm{C}$, Hatsukami DK, et al. Smokeless tobacco, snus, at admission for percutaneous coronary intervention and future risk for cardiac events. Open Heart 2019;6:e001109. doi:10.1136/ openhrt-2019-001109

Received 10 June 2019 Revised 18 September 2019 Accepted 23 September 2019

Check for updates

C) Author(s) (or their employer(s)) 2019. Re-use permitted under CC BY-NC. No commercial re-use. See rights and permissions. Published by BMJ.

${ }^{1}$ Faculty of Health, Department of Cardiology, Örebro University, Örebro, Sweden

${ }^{2}$ Faculty of Medicine, Department of Cardiology, Lund University, Lund, Sweden ${ }^{3}$ Department of Psychiatry, Tobacco Research Programs, University of Minnesota, Minneapolis, Minnesota, USA ${ }^{4}$ Unit of Cardiology, Department of Medicine, Heart and Vascular Division, Karolinska Institutet, Stockholm, Sweden ${ }^{5}$ Department of Cardiology, Sahlgrenska University Hospital, Gothenburg, Sweden

${ }^{6}$ Unit of Cardiology, Department of Medicine, Heart and Vascular Division, Karolinska Institutet, Stockholm, Sweden

Correspondence to Dr Ole Frobert; ole.frobert@ regionorebrolan.se

\section{ABSTRACT}

Objective To assess the risk of future death and cardiac events following percutaneous coronary intervention (PCI) in patients using smokeless tobacco, snus, compared with patients not using snus at admission for a first PCl.

Methods The Swedish Coronary Angiography and Angioplasty Registry is a prospective registry on coronary diagnostic procedures and interventions. A total of 74958 patients admitted for a first $\mathrm{PCl}$ were enrolled between 2009 and 2018, 6790 snus users and 68168 not using snus. We used Cox proportional hazards regression for statistical modelling on imputed datasets as well as complete-case datasets.

Results Patients using snus were younger (mean (SD) age $61.0( \pm 10.2)$ years) than patients not using snus $(67.6$ $( \pm 11.1), \mathrm{p}<0.001)$ and more often male $(95.4 \%$ vs $67.4 \%$, $\mathrm{p}<0.001)$. After multivariable adjustment, snus use was not associated with the primary composite outcome of all-cause mortality, new coronary revascularisation or new hospitalisation for heart failure at 1 year $(\mathrm{HR} 0.98,95 \% \mathrm{Cl}$ 0.91 to 1.05). In patients using snus at baseline who underwent a second $\mathrm{PCl}(\mathrm{n}=1443)$, the duration from the index intervention was shorter for subjects who continued using snus ( $n=921,63.8 \%$ ) compared with subjects who had stopped (mean number of days 285 vs 406, $p$ value $=0.001$ )

Conclusions Snus use at admission for a first $\mathrm{PCl}$ was not associated with a higher occurrence of all-cause mortality, new revascularisation or heart failure hospitalisation. Discontinuing snus after a first PCI was associated with a significantly longer duration to a subsequent $\mathrm{PCl}$.

\section{BACKGROUND}

The risk of cardiovascular disease (CVD) is lower with smokeless tobacco than with cigarettes. ${ }^{1}$ Yet smokeless tobacco use is discouraged by the American Heart Association because of evidence indicating increased risks of fatal myocardial infarction (MI) and fatal stroke and poorer prognosis after MI and stroke. ${ }^{2}$

Swedish snus is a dry form of moist snuff sold as sachets or in loose form in small plastic or tin cans. In contrast to (American) snuff, snus is placed in the upper lip (not the lower) and is air cured and sun cured (not

\section{Key questions}

What is already known about this subject?

- The use of smokeless tobacco is on the rise, but information on cardiac risk is limited.

- Most previous studies have not demonstrated increased cardiovascular risk in subjects using snus, but little is known of risks after a cardiac event.

What does this study add?

- In this cohort study covering all patients undergoing a first percutaneous coronary intervention (PCI) in Sweden over 9 years, snus use was not associated with a higher occurrence of all-cause mortality, new revascularisation or heart failure at 1 year.

- Importantly, discontinuing snus after a first PCI was associated with a longer duration to a subsequent $\mathrm{PCl}$.

How might this impact on clinical practice?

- The harmful effects from snus are less than found with smoking but not negligible.

- Our findings can be used to inform patients and health professionals about the health risks of smokeless tobacco.

fire cured) and pasteurised, which reduces the levels of harmful chemicals and used typically for 11-14 hours per day. ${ }^{1}$

Although the association between smoking and CVD risk is well established, the effects of snus on CVD risk is less certain. One prospective Swedish cohort study found that continuing snus users had a higher mortality rate than did snus quitters after an $\mathrm{MI}^{3}{ }^{3} \mathrm{~A}$ twin cohort study involving more than 16000 men found no association between snus use and CVD risk, ${ }^{4}$ and a pooled analysis of observational studies could not demonstrate a higher MI risk with snus use. ${ }^{5}$ Snus use was not found to be associated with stroke ${ }^{6}$ or atrial fibrillation, ${ }^{7}$ while there could be an association between the use of snus and heart failure. ${ }^{8}$

The Swedish Coronary Angiography and Angioplasty Registry (SCAAR) holds data on consecutive patients from all 30 centres 
that perform coronary angiography and percutaneous coronary intervention (PCI) in Sweden. From 2009 and onwards, active or previous use of snus has been registered along with active or previous smoking. In previous studies of snus use in Sweden, no association with increased cardiovascular risk was found. ${ }^{9}$ Our objective was to investigate the risk of all-cause death or new revascularisation with snus use at admission in patients undergoing PCI and registered in SCAAR. We hypothesised that active use of snus confers an increased risk of future CVD events.

\section{METHODS}

\section{Study population}

Our study included all patients $\geq 18$ years of age in Sweden who underwent a first PCI from 1 January 2009 to 31 May 2018 at all hospitals performing PCI with the indications stable angina, unstable angina, non-ST-segment elevation MI (NSTEMI) or ST-segment elevation MI. Patients who had a history of previous MI or who had previously been treated with PCI or coronary artery by-pass surgery were excluded from this report. We also excluded patients for whom information on the use of snus was unavailable and those who did not have complete follow-up data. The application of inclusion and exclusion criteria resulted in a total of 74958 individuals eligible for analysis (figure 1).

We performed additional analyses to investigate longitudinal use of snus $(n=6790)$ and assess whether the discontinuation of snus after the index PCI and a subsequent PCI was associated with the primary and secondary outcomes (see online supplementary figure S1).

\section{The SCAAR data}

SCAAR, which is a part of the national Swedish Web System for Enhancement and Development of Evidence-based Care in Heart Disease Evaluated According to Recommended Therapies registry, holds data on all consecutive patients from all 30 centres that perform coronary angiography and PCI in Sweden. The registry is sponsored by the Swedish Health Authorities and is independent of commercial funding. The technology is developed and administered by the Uppsala Clinical Research Center. Since 2001, SCAAR has been internet based, with recording of data online through a web interface in the catheterisation laboratory; data are transferred in an encrypted format to a central server at the Uppsala Clinical Research Center. Long-term follow-up was obtained by merging the SCAAR database with other national registries based on all Swedish citizens' unique personal identification number. Vital status and date of death was obtained from the National Population registry and was available until 20 May 2018. Because the data are anonymised, written informed consent from each patient was not needed;

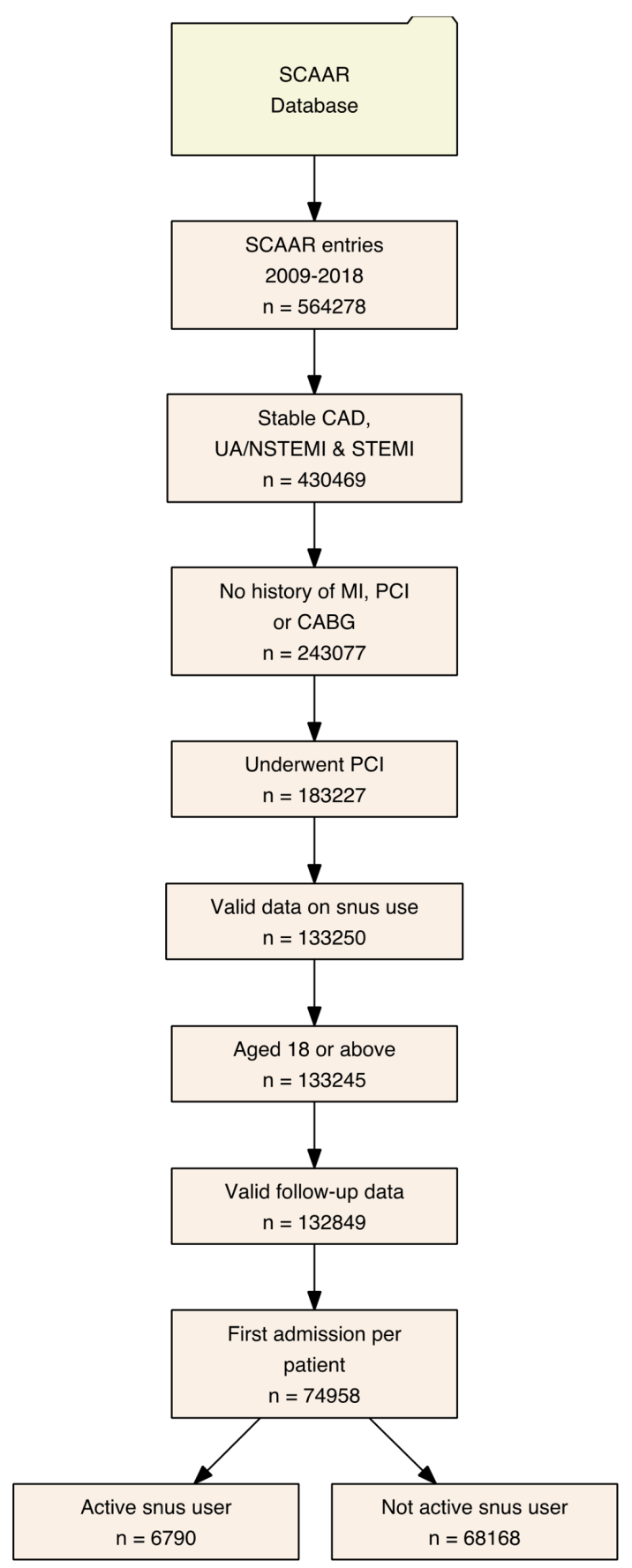

Figure 1 Flow chart of study design, inclusion and exclusion criteria and final study population. CAD, coronary artery disease; CABG, coronary artery bypass grafting; $\mathrm{MI}$, myocardial infarction; $\mathrm{PCl}$, percutaneous coronary intervention; SCAAR, Swedish Coronary Angiography and Angioplasty Registry; STEMI, ST-segment elevation MI; UA/ NSTEMI, unstable angina/non-ST-segment elevation MI.

however, patients can choose to opt out of the registry at any time. Monitoring and verification of registry data have been performed in all hospitals since 2001 by comparing 50 entered variables in 20 randomly selected interventions per hospital and year with the patients' hospital records. The overall correspondence of data during the study period was $>95 \% .{ }^{10}$ In Sweden, patient-reported smoking status and use of 
Interventional cardiology

Table 1 Patient characteristics

\begin{tabular}{|c|c|c|c|c|}
\hline & Not using snus $(n=68168)$ & Using snus $(n=6790)$ & Missing (\%) & $P$ value \\
\hline \multicolumn{5}{|l|}{ Demographics } \\
\hline Age & $67.6( \pm 11.1)$ & $61.0( \pm 10.2)$ & 0.0 & $<0.001$ \\
\hline Male & $45397(67.4)$ & $6476(95.4)$ & 0.0 & $<0.001$ \\
\hline Body mass index & $27.3( \pm 6.0)$ & $28.3( \pm 5.8)$ & 11.1 & $<0.001$ \\
\hline Never smoked & $31251(46.2)$ & $1674(24.9)$ & & \\
\hline Former smoker & $22824(33.8)$ & $3353(49.9)$ & & \\
\hline Current smoker & $13515(20.0)$ & $1693(25.2)$ & & \\
\hline \multicolumn{5}{|l|}{ Comorbidities } \\
\hline Diabetes & $13117(19.3)$ & $1201(17.7)$ & 0.1 & 0.002 \\
\hline Hyperlipidaemia & $22767(33.6)$ & $2117(31.3)$ & 0.6 & $<0.001$ \\
\hline Estimated GFR & $78.3( \pm 18.8)$ & $85.3( \pm 17.1)$ & 27.0 & $<0.001$ \\
\hline CKD stage & & & 27.0 & $<0.001$ \\
\hline Chronic kidney disease stage I-II & $41571(83.4)$ & $4485(91.9)$ & & \\
\hline Chronic kidney disease stage III & $7558(15.2)$ & $358(7.3)$ & & \\
\hline Chronic kidney disease stage IV-V & $723(1.5)$ & $36(0.7)$ & & \\
\hline COPD & $3167(4.6)$ & $286(4.2)$ & 0 & 0.111 \\
\hline Peripheral artery disease & $1700(2.5)$ & $151(2.2)$ & 0 & 0.185 \\
\hline Previous stroke & $3366(4.9)$ & $227(3.3)$ & 0 & $<0.001$ \\
\hline Indication & & & 0.0 & 0.016 \\
\hline Stable CAD & $12742(18.7)$ & $1178(17.3)$ & & \\
\hline UA/NSTEMI & $34056(50.0)$ & $3485(51.3)$ & & \\
\hline STEMI & $21370(31.3)$ & $2128(31.3)$ & & \\
\hline \multicolumn{5}{|l|}{ Medical treatments } \\
\hline Upstream DAPT & $54149(79.4)$ & $5479(80.7)$ & 0.0 & 0.014 \\
\hline Aspirin & 66551 (97.6) & $6651(98.0)$ & 0.0 & 0.091 \\
\hline Clopidogrel & $29115(42.7)$ & $2609(38.4)$ & 0.0 & $<0.001$ \\
\hline Prasugrel & $1452(2.1)$ & $202(3.0)$ & 0.0 & $<0.001$ \\
\hline Ticagrelor & $36674(53.8)$ & $3925(57.8)$ & 0.0 & $<0.001$ \\
\hline Heparin & 60732 (89.1) & $6169(90.9)$ & 0.0 & $<0.001$ \\
\hline Bivalirudin & $17388(25.5)$ & $1728(25.4)$ & 0.0 & 0.916 \\
\hline LMWH & 3529 (5.2) & $268(3.9)$ & 0.0 & $<0.001$ \\
\hline Fondaparinux & $18258(26.8)$ & $1941(28.6)$ & 0.0 & 0.001 \\
\hline \multicolumn{5}{|l|}{ Procedure characteristics } \\
\hline Vascular approach & & & 0.0 & $<0.001$ \\
\hline Femoral & $9200(13.5)$ & $796(11.7)$ & & \\
\hline
\end{tabular}


Table 1 Continued

\begin{tabular}{|c|c|c|c|c|}
\hline & Not using snus $(n=68168)$ & Using snus $(n=6790)$ & Missing (\%) & $P$ value \\
\hline Other & 58967 (86.5) & $5994(88.3)$ & & \\
\hline Diseased vessels & & & 0.4 & 0.001 \\
\hline Two & $19990(29.5)$ & $1924(28.5)$ & & \\
\hline Three & $9918(14.6)$ & $898(13.3)$ & & \\
\hline Stented & $59207(86.9)$ & $5986(88.2)$ & 0.0 & 0.002 \\
\hline Drug-eluting stent & 48660 (82.2) & $5052(84.4)$ & & $<0.001$ \\
\hline Complete revascularisation & $4280(69.7)$ & $4574(73.5)$ & 8.8 & $<0.001$ \\
\hline
\end{tabular}

CAD, coronary artery disease; CKD, chronic kidney disease according to the CKD-EPI formula; COPD, chronic obstructive pulmonary disease; DAPT, dual antiplatelet therapy; GFR, glomerular filtration rate; LMWH, low molecular weight heparin; STEMI, ST-segment elevation myocardial infarction; UA/NSTEMI, unstable angina/non-ST-segment elevation myocardial infarction.

snus is obtained at the time of coronary angiography and registered in SCAAR by the treating interventionist.

\section{Outcomes}

The primary composite outcome of this study was a first occurrence of either all-cause death, new coronary revascularisation (defined as a planned or unplanned coronary investigation with an angiogram warranting intervention with PCI or coronary artery bypass surgery) or new hospitalisation for heart failure. Secondary outcome measures were the individual components of the primary composite outcome and time to new coronary intervention.

\section{Statistics}

Continuous variables are expressed as means \pm SD. Categorical variables are expressed as counts and percentages. Differences in continuous variables between snus users and those not using snus were assessed with

\begin{tabular}{|llllc}
\hline Table 2 Outcomes & & & & \\
\hline & HR & Cl (lower) & Cl (upper) & P value \\
\hline Primary composite outcome & & & & \\
\hline Univariable & 0.824 & 0.772 & 0.88 & $<0.001$ \\
\hline Age and sex adjusted & 0.969 & 0.907 & 1.036 & 0.359 \\
\hline Multivariable, imputed & 0.978 & 0.914 & 1.045 & 0.508 \\
\hline Multivariable, complete case & 0.975 & 0.896 & 1.06 & 0.554 \\
\hline All-cause mortality & & & & \\
\hline Univariable & 0.639 & 0.548 & 0.746 & $<0.001$ \\
\hline Age and sex adjusted & 1.155 & 0.987 & 1.352 & 0.072 \\
\hline Multivariable, imputed & 1.146 & 0.979 & 1.342 & 0.09 \\
\hline Multivariable, complete case & 1.17 & 0.954 & 1.434 & 0.132 \\
\hline New revascularisation & & & & \\
\hline Univariable & 0.996 & 0.917 & 1.081 & 0.915 \\
\hline Age and sex adjusted & 0.937 & 0.862 & 1.018 & 0.123 \\
\hline Multivariable, imputed & 0.941 & 0.866 & 1.023 & 0.154 \\
\hline Multivariable, complete case & 0.926 & 0.835 & 1.028 & 0.148 \\
\hline Heart failure hospitalisation & & & & \\
\hline Univariable & 0.623 & 0.548 & 0.709 & $<0.001$ \\
\hline Age and sex adjusted & 0.912 & 0.800 & 1.039 & 0.167 \\
\hline Multivariable, imputed & 0.915 & 0.802 & 1.043 & 0.183 \\
\hline Multivariable, complete case & 0.973 & 0.826 & 1.147 & 0.745 \\
\hline
\end{tabular}

The first model is unadjusted, the second model adjusted for age and sex and the third model is the full multivariable model. The multivariable models are shown both pooled from analyses on the multiply imputed dataset and complete case only dataset. 
A

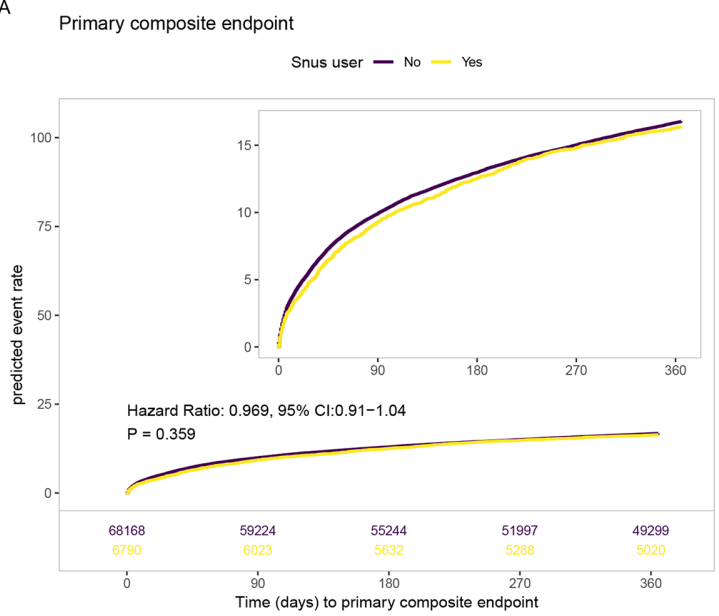

$\mathrm{C}$

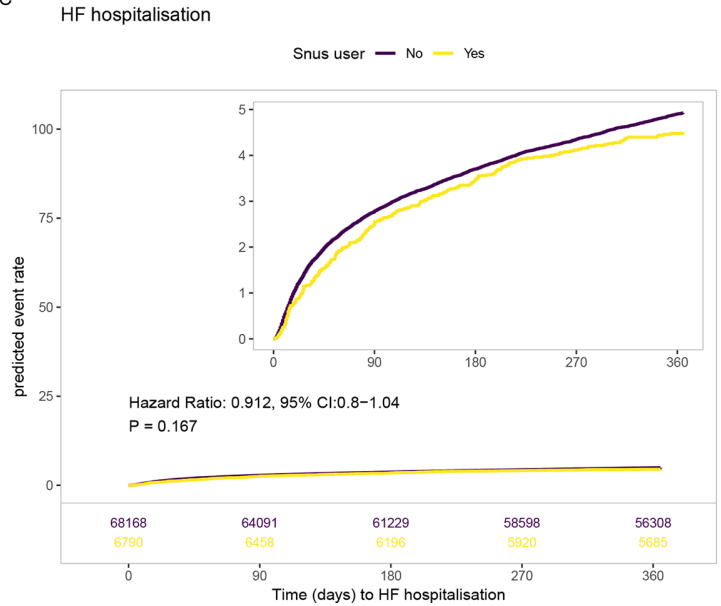

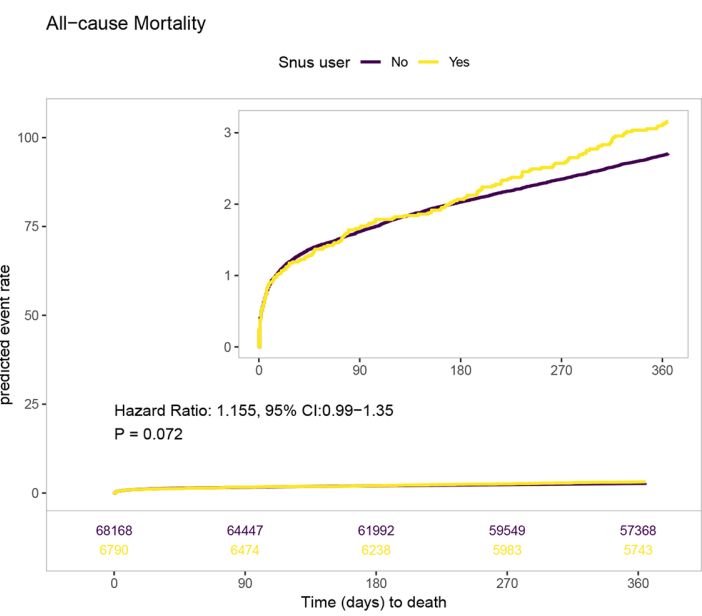

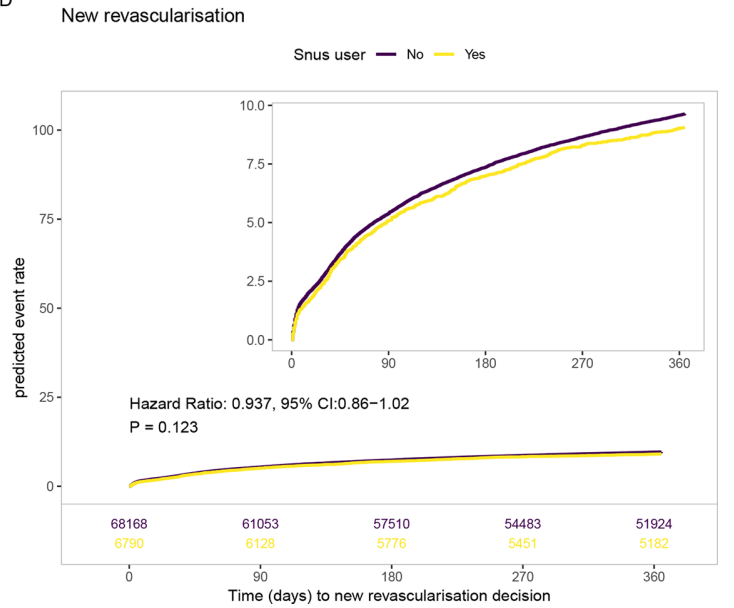

Figure 2 Survival curves for (A) the primary composite outcome of all-cause death, new coronary revascularisation and new hospitalisation for heart failure (HF); (B) all-cause death; (C) new revascularisation; and (D) new hospitalisation for HF. The curves are estimated from Cox proportional hazards models adjusting for age and sex. The $p$ values are derived from the corresponding Cox proportional hazards models.

Student's t-test. Differences between categorical variables were assessed with the $\chi^{2}$ test. Independent associations between snus use and age, sex, body mass index and other covariables were calculated with multivariable logistic regression. Univariable and multivariable HRs with 95\% CIs as well as age-adjusted and sex-adjusted survival graphs were calculated with Cox proportional hazards models. Model 1 included only current snus use as independent variable; model 2 adjusted for age (modelled as restricted cubic spline) and sex; model 3 accounted for age, sex, body mass index, smoking status and comorbidities (diabetes, hypertension, hyperlipidaemia, renal function (estimated glomerular filtration rate (eGFR)), chronic obstructive pulmonary disease, peripheral artery disease, previous stroke, cancer and heart failure). Percentage of missing values is shown for each variable and was low apart from body mass index $(11.1 \%)$ and eGFR $(27.0 \%)$. Missing data, which were assumed to be missing at random, were imputed using multiple imputation (five imputed datasets, all baseline characteristics and outcome covariables were used to predict missing values). Results from analyses on each imputed data set were then pooled according to Rubin's rules. ${ }^{11}$ The variables for snus use, age and sex (ie, those included in models 1 and 2) did not include any missing values. Results from multivariable models including only complete cases $(n=51409)$ for all four outcome measures were reported for consistency. For the multivariable models (model 3 and the complete cases model), HRs for smokers versus non-smokers were also reported. Predefined subgroup analyses and interaction tests were performed on complete cases in men versus women, patients currently versus not currently smoking, in patients with versus without diabetes mellitus, in patients with versus without hypertension and in patients with versus without renal dysfunction (eGFR $<60 \mathrm{~mL}$ / $\min / 1.73 \mathrm{~m}^{2}$ ). The subgroup analyses were adjusted for the same variables as the multivariable model 3 .

In a longitudinal analysis of snus use and subsequent PCI, we investigated those who were using snus at the index PCI $(\mathrm{n}=6790)$ and underwent a subsequent second PCI during the study time. We descriptively analysed the 


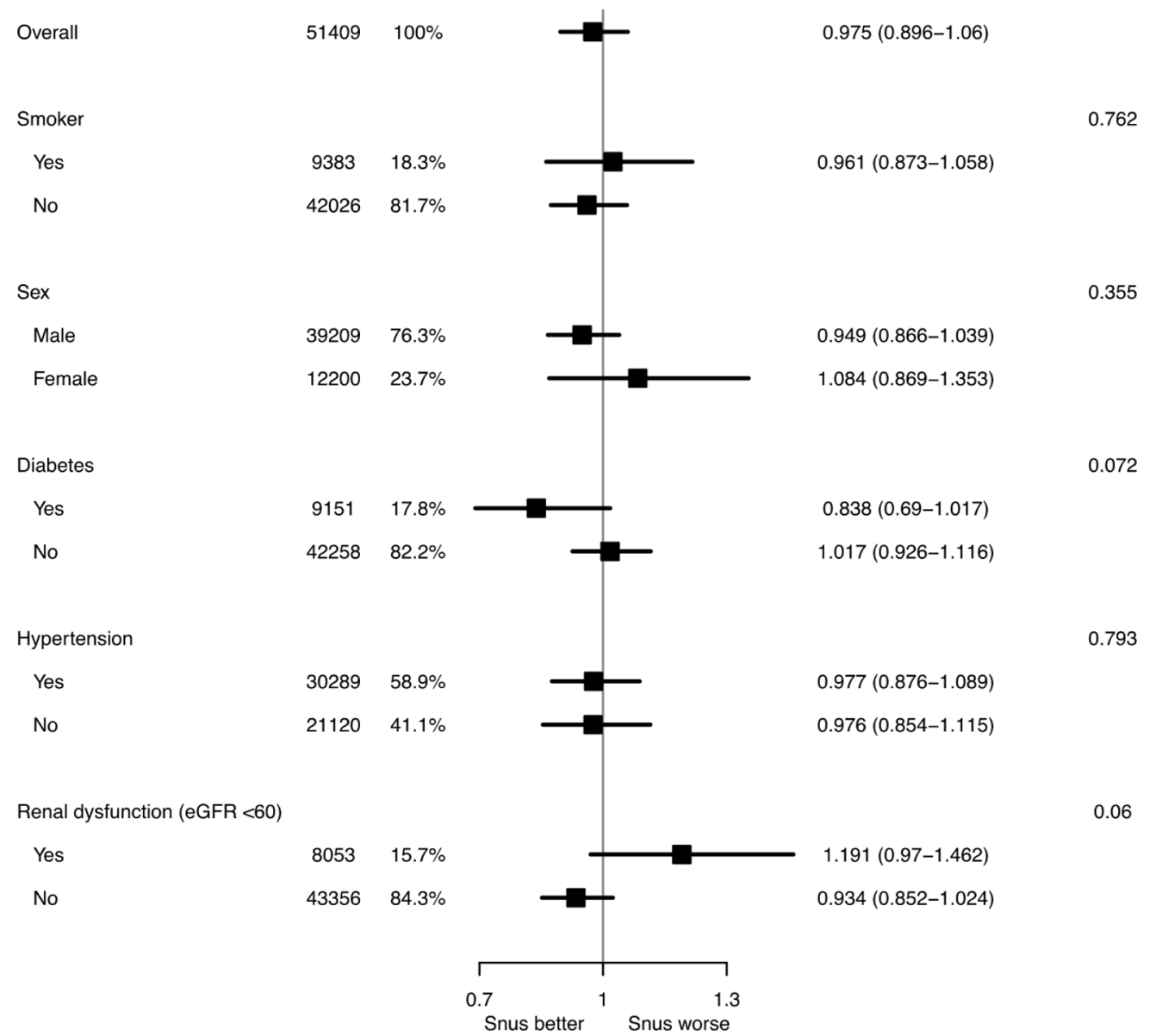

Figure 3 Forest plot displaying HRs and 95\% Cls for subgroups and their interaction $\mathrm{p}$ values. The plot was produced with pooled results from multivariable analyses of the imputed data sets on the primary composite outcome. The analyses are multivariable and adjusted as in model 3. eGFR, estimated glomerular filtration rate.

proportion of snus users who continued to use or discontinued snus at subsequent PCIs during the follow-up, and we calculated the mean time from the first to the second PCI stratified by those who had stopped or continued using snus, respectively. We also investigated the association between discontinuing snus and outcomes using univariable as well as age-adjusted and sex-adjusted Cox proportional hazards models.

Statistical analyses were performed in R (version 3.5.1, R Foundation for Statistical Computing, Vienna, Austria). All $p$ values are two tailed, and a value of 0.05 was considered statistically significant.

\section{RESULTS}

\section{Patient characteristics}

Baseline and procedural characteristics are shown in table 1. We identified a total of 6790 current snus users and 68168 subjects not using snus. Of note, snus users were younger (mean 61.0 vs 67.6 years), more often of male sex, had a higher body mass index and were more often current or previous smokers than subjects not using snus. However, diabetes mellitus, hypertension and hyperlipidaemia were less frequent in snus users who also had better renal function. Snus users had less advanced coronary artery disease and were more often completely revascularised compared with patients not using snus. The temporal trend of snus use showed an increase from around $6 \%-9 \%$ of patients in the first few years (20092011) after which snus use plateaued (see online supplementary figure S2).

We found independent positive associations between snus use (see online supplementary table S1) and younger age, male sex, high body mass index, previous or active smoking, late study inclusion and presentation with unstable angina/NSTEMI.

\section{Outcome}

In the unadjusted analyses (model 1), snus use was significantly associated with better outcome of the primary composite outcome, all-cause mortality and heart failure hospitalisation analyses compared with not using snus (table 2). After adjusting for age and sex (model 2), snus use was not associated with the primary composite outcome or the secondary outcomes (figure 2). After 


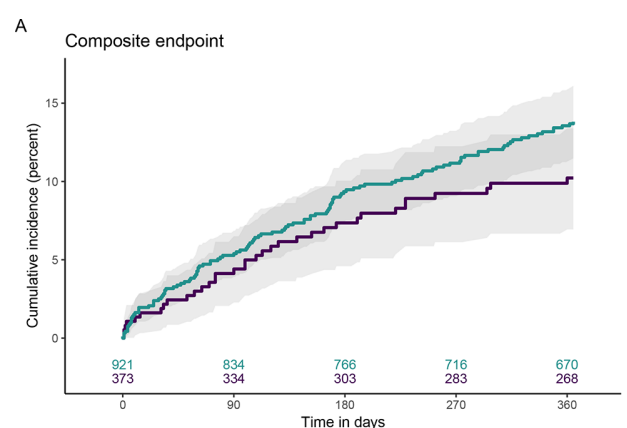

C

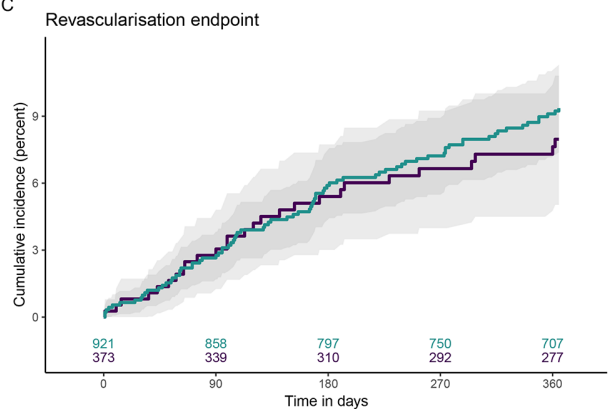

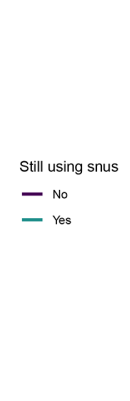

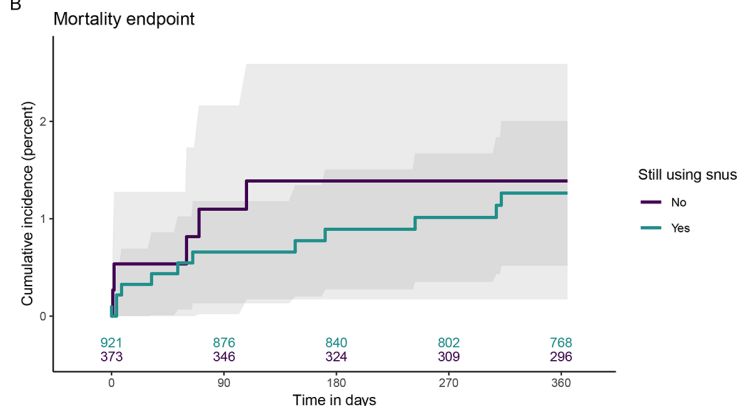

D

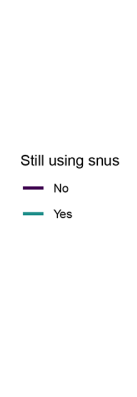

D

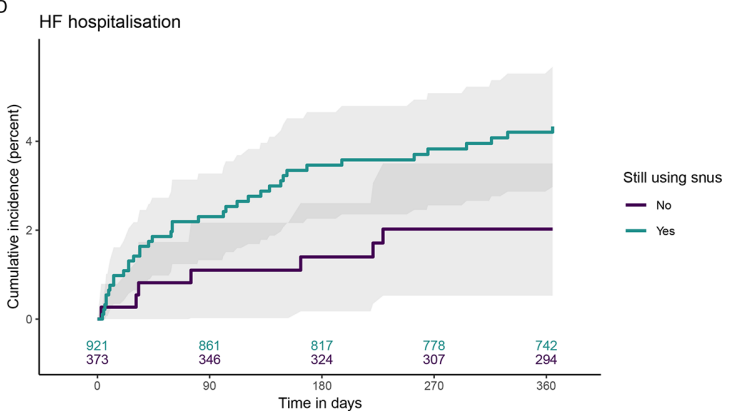

Figure 4 Outcomes in snus users and snus quitters following the index $\mathrm{PCl}$. Unadjusted Kaplan-Meier graphs for (A) the primary composite endpoint of all-cause death, new coronary revascularisation and new hospitalisation for heart failure; (B) all-cause death; (C) new revascularisation; and (D) new hospitalisation for heart failure (HF). Only patients who used snus at the index $\mathrm{PCl}$ are included. $\mathrm{PCl}$, percutaneous coronary intervention.

applying the additional adjustments for differences in body mass index, smoking status and comorbidities, there were no significant associations between snus use and any outcome, both in the analyses using multiply imputed data and those performed on complete cases.

\section{Subgroup analyses}

There were no significant interactions between snus use and the investigated subgroups. Of note the HR of the primary composite outcome in patients using snus who had an eGFR $<60 \mathrm{~mL} / \mathrm{min}$ was 1.191 (95\% CI 0.97 to 1.46) compared with those with a higher eGFR (HR 0.93, $95 \%$ CI 0.85 to 1.02 , $\mathrm{p}$ value for interaction $=0.06$ ). Snus use in the subpopulation with diabetes was associated with a HR of 0.838 (95\% CI 0.69 to 1.02) compared with those with no diabetes (HR 1.02, 95\% CI 0.93 to 1.12, p value for interaction $=0.07$ ) (figure 3 ).

\section{Outcome for smokers}

Smokers had a significantly higher risk of experiencing the primary composite outcome, all-cause mortality and heart failure hospitalisation compared with non-smokers (HRs in model 3: 1.201, 1.742 and 1.502, respectively, all $\mathrm{p}$ values $<0.001)$. Smoking was not associated with the revascularisation outcome ( $\mathrm{p}$ value 0.75 in model 3) (see online supplementary table S2).

\section{Snus use following the index PCI}

Among the 6790 patients who used snus at the index PCI, 1443 underwent a subsequent second PCI during the study period. These patients were stratified according to their snus use status at the time of the second PCI into a group that had continued to use snus $(\mathrm{n}=921(63.8 \%))$ and a group that had discontinued snus (n=373 (25.8\%)) (see online supplementary figure S1). Data on snus use were missing for 149 patients $(10.4 \%)$. Baseline characteristics for these groups were well balanced (see online supplementary table S3). The proportion of patients using snus and/or smoking decreased at subsequent PCI procedures in this subpopulation (see online supplementary figure S3 and S4). In patients who underwent a second PCI, the duration from the index intervention was shorter for subjects who continued using snus compared with subjects who had stopped (mean number of days 285 vs $406, p$ value $=0.001$ ). After undergoing a second PCI, there were no significant associations between continued snus use and worse outcome at 1 year, but cases were few. The primary composite outcome (age and sex adjusted) HR was 1.36 (95\% CI 0.94 to $1.99, \mathrm{p}=0.107$ ) and for heart failure hospitalisation (age and sex adjusted) HR was 2.20 (95\% CI 0.98 to $4.93, \mathrm{p}=0.055$ ) in patients who reported still using snus at the subsequent PCI following the index PCI (see online supplementary table S4, figure 4).

\section{DISCUSSION}

In this study covering more than 9 years and all invasive coronary centres in Sweden, our main findings after statistical adjustment for known confounders were that snus use at admission for a first PCI was not associated with a higher occurrence of the combined outcome of all-cause mortality, new revascularisation or heart failure hospitalisation at one year. Discontinuing snus after a first PCI was associated with a significantly longer duration to a subsequent PCI. 
A recent comprehensive meta-analysis on smokeless tobacco use identified 11 cohort studies conducted in Sweden and 6 in the USA. ${ }^{9}$ The analysis did not show increased risk of heart disease or stroke among snus users in Sweden compared with non-users but demonstrated increased heart disease and stroke risk for smokeless tobacco users in the USA. As pointed out by the authors, US smokeless tobacco products contain higher levels of nicotine, tobacco-specific nitrosamines including carcinogens, nitrite, nitrate and chloride and polycyclic aromatic hydrocarbons compared with Swedish snus, ${ }^{12} 13$ and Swedish snus has lower levels of acrolein, a substance associated with oxidative stress and endothelial dysfunction. ${ }^{13}$ While snus use in Sweden may not be associated with increased cardiovascular risk, the focus of our study was to investigate the prognostic implication of snus use at admission for CVD-an area with a paucity of studies. We identified one prospective Swedish cohort study showing that continuing snus users had a higher mortality rate than did snus quitters after an MI. ${ }^{3}$ While that study used a clinical definition of MI and followed patients from two months after diagnosis, we used angiographically established coronary artery disease warranting PCI as inclusion criterion and followed patients from time of diagnosis. Furthermore, our study sample was more than thrice that of the previous study.

A policy statement from the American Heart Association concluded that nicotine may contribute to the effects of smoking on cardiovascular health but that other constituents in cigarette smoke seem to have much more important deleterious effects. ${ }^{1}$ We found that smokers had a worse outcome across all outcomes in adjusted analyses except revascularisation, while this was not the case for snus users. It is probably safe to say that the primary harmful substance in tobacco is nicotine. Nicotine induces strong sympathetic stimulation, ${ }^{14}$ elevating blood pressure ${ }^{15}$ and heart rate. ${ }^{16}$ Nicotine deteriorates arterial compliance acutely ${ }^{17}$ induces endothelial dysfunction ${ }^{18}$ and results in increased arterial stiffness in the long term. ${ }^{19}$ In an animal model, nicotine impaired myocardial healing and altered left ventricular remodelling after $\mathrm{MI},{ }^{20}$ and in a human cohort study, snus users had an elevated risk of heart failure. ${ }^{8}$ Platelet responsiveness may also be affected by nicotine, ${ }^{21}$ while coagulation indices seem unaffected. ${ }^{22}$ Inflammation is central in the development of coronary artery disease ${ }^{23}$ and in-stent restenosis ${ }^{24}$ and while there is broad agreement that cigarette smoke is proinflammatory, ${ }^{25}$ information on snus use and inflammation is limited. In our study, we observed a shorter duration to subsequent PCI in snus users who continued to use compared with quitters. These findings support the conclusion that snus is not safe; however, the harmful effects from snus are less than found with smoking.

\section{Strengths and limitations}

Strengths to our study include a large real-world patient population with data from all invasive coronary procedures in an entire country over more than 9 years with a high degree of accuracy. As with virtually all observational studies, there are, however, limitations, despite use of prospectively recorded data from national registries with a high level of completeness. The validity of variables recorded in SCAAR is high and loss to follow-up is low, ${ }^{10}$ but as misclassification occurs, this reduces precision of our findings. Additionally, residual confounding is always a possibility in observational studies. For example, we had no data on socioeconomic status, which is associated with unhealthy behaviours and the socioeconomic gradient in CVD and mortality. ${ }^{26}$ An additional limitation to our study is that we did not have access to snus use data after the index PCI except for patients undergoing subsequent PCI procedures, and in those who had discontinued snus, we had no information on when they had stopped. Snus sold in Sweden typically has a nicotine content between $8 \mathrm{mg} / \mathrm{g}$ and $14 \mathrm{mg} / \mathrm{g}$ tobacco, but extra strong varieties with a nicotine content of $45 \mathrm{mg} / \mathrm{g}$ are also available (information accessed at www.swedishmatch.se). Type of snus used is not registered in SCAAR, which is a study limitation.

In conclusion, snus use at admission for a first PCI was not associated with a higher occurrence of the combined outcome of all-cause mortality, new revascularisation or heart failure hospitalisation at 1 year. Although most previous studies on use of Swedish snus did not find an association with increased future cardiovascular risk, our data indicate that snus at the time of angiographic diagnosis and continuing snus use thereafter is associated with a significantly shorter duration to a subsequent PCI.

\section{Twitter Ole Frobert @Frobert0le}

Contributors OF, CR and PA conceptualised the study. CR and PA performed statistical analyses. OF wrote initial manuscript draft. CR, DKH, JP, EO and PA commented on manuscript and revisions.

Funding The authors have not declared a specific grant for this research from any funding agency in the public, commercial or not-for-profit sectors.

Competing interests None declared.

Patient consent for publication Not required.

Provenance and peer review Not commissioned; externally peer reviewed.

Data availability statement Data may be obtained from a third party and are not publicly available.

Open access This is an open access article distributed in accordance with the Creative Commons Attribution Non Commercial (CC BY-NC 4.0) license, which permits others to distribute, remix, adapt, build upon this work non-commercially, and license their derivative works on different terms, provided the original work is properly cited, appropriate credit is given, any changes made indicated, and the use is non-commercial. See: http://creativecommons.org/licenses/by-nc/4.0/.

ORCID iD

Ole Frobert http://orcid.org/0000-0002-5846-345X

\section{REFERENCES}

1 Piano MR, Benowitz NL, Fitzgerald GA, et al. Impact of smokeless tobacco products on cardiovascular disease: implications for policy, prevention, and treatment: a policy statement from the American heart association. Circulation 2010;122:1520-44.

2 Henley SJ, Thun MJ, Connell C, et al. Two large prospective studies of mortality among men who use snuff or chewing tobacco (United States). Cancer Causes Control 2005;16:347-58. 
3 Arefalk G, Hambraeus K, Lind L, et al. Discontinuation of smokeless tobacco and mortality risk after myocardial infarction. Circulation 2014;130:325-32.

4 Hansson J, Pedersen NL, Galanti MR, et al. Use of snus and risk for cardiovascular disease: results from the Swedish twin registry. $J$ Intern Med 2009;265:717-24.

5 Hansson J, Galanti MR, Hergens M-P, et al. Use of snus and acute myocardial infarction: pooled analysis of eight prospective observational studies. Eur J Epidemiol 2012;27:771-9.

6 Hansson J, Galanti MR, Hergens M-P, et al. Snus (Swedish smokeless tobacco) use and risk of stroke: pooled analyses of incidence and survival. J Intern Med 2014;276:87-95.

7 Hergens M-P, Galanti R, Hansson J, et al. Use of Scandinavian moist smokeless tobacco (snus) and the risk of atrial fibrillation. Epidemiology 2014;25:872-6.

8 Arefalk G, Hergens M-P, Ingelsson E, et al. Smokeless tobacco (snus) and risk of heart failure: results from two Swedish cohorts. Eur J Prev Cardiol 2012;19:1120-7.

9 Rostron BL, Chang JT, Anic GM, et al. Smokeless tobacco use and circulatory disease risk: a systematic review and meta-analysis. Open Heart 2018;5:e000846.

10 Fröbert O, Lagerqvist B, Olivecrona GK, et al. Thrombus aspiration during ST-segment elevation myocardial infarction. N Engl J Med 2013;369:1587-97.

11 Buuren Svan, Groothuis-Oudshoorn K. mice: Multivariate Imputation by Chained Equations in R. J Stat Softw 2011;45:1.67.

12 Stepanov I, Jensen J, Hatsukami D, et al. New and traditional smokeless tobacco: comparison of toxicant and carcinogen levels. Nicotine Tob Res 2008;10:1773-82.

13 Borgerding MF, Bodnar JA, Curtin GM, et al. The chemical composition of smokeless tobacco: a survey of products sold in the United States in 2006 and 2007. Regul Toxicol Pharmacol 2012;64:367-87.

14 Johnston R, Crowe M, Doma K. Effect of nicotine on repeated bouts of anaerobic exercise in nicotine naïve individuals. Eur J Appl Physiol 2018;118:681-9.
15 Van Slyke CB, Larson PS. Observations on the role of the adrenal medulla in the blood pressure response to nicotine. J Pharmacol Exp Ther 1950;98:400-4.

16 Perkins KA, Sexton JE, Reynolds WA, et al. Comparison of acute subjective and heart rate effects of nicotine intake via tobacco smoking versus nasal spray. Pharmacol Biochem Behav 1994:47:295-9

17 Adamopoulos D, Argacha J-F, Gujic M, et al. Acute effects of nicotine on arterial stiffness and wave reflection in healthy young non-smokers. Clin Exp Pharmacol Physiol 2009;36:784-9.

18 Rohani M, Agewall S. Oral snuff impairs endothelial function in healthy snuff users. J Intern Med 2004:255:379-83.

19 Wagenhäuser MU, Schellinger IN, Yoshino T, et al. Chronic nicotine exposure induces murine aortic remodeling and stiffness Segmentation-Implications for abdominal aortic aneurysm susceptibility. Front Physiol 2018;9:1459.

20 Villarreal FJ, Hong D, Omens J. Nicotine-modified postinfarction left ventricular remodeling. Am J Physiol 1999;276:H1103-6.

21 Whiss PA, Lundahl TH, Bengtsson T, et al. Acute effects of nicotine infusion on platelets in nicotine users with normal and impaired renal function. Toxicol Appl Pharmacol 2000;163:95-104.

22 Blann AD, Steele C, McCollum CN. The influence of smoking and of oral and transdermal nicotine on blood pressure, and haematology and coagulation indices. Thromb Haemost 1997;78:1093-6.

23 Libby P, Tabas I, Fredman G, et al. Inflammation and its resolution as determinants of acute coronary syndromes. Circ Res 2014;114:1867-79.

24 Kornowski R, Hong MK, Tio FO, et al. In-stent restenosis: contributions of inflammatory responses and arterial injury to neointimal hyperplasia. J Am Coll Cardiol 1998;31:224-30.

25 Bhalla DK, Hirata F, Rishi AK, et al. Cigarette smoke, inflammation, and lung injury: a mechanistic perspective. J Toxicol Environ Health $B$ Crit Rev 2009;12:45-64.

26 Petrovic D, de Mestral C, Bochud M, et al. The contribution of health behaviors to socioeconomic inequalities in health: a systematic review. Prev Med 2018;113:15-31. 